\title{
Research Situation and Applications of Intelligent Materials
}

\author{
Shang Miao \\ College of Mechanical Engineering \\ Xijing University \\ Xi'an, China \\ e-mail: 445700839@qq.com \\ Lin Guomin \\ College of Mechanical Engineering \\ Xijing University \\ Xi'an, China \\ e-mail: 641922530@qq.com
}

\author{
Sun Yongtao \\ Avic xi 'an aircraft industry \\ Xi'an, China \\ e-mail: 258510717@qq.com
}

\author{
Wang YongFu \\ College of Mechanical Engineering \\ Xijing University \\ Xi'an, China \\ e-mail: 1137919199@qq.com
}

\begin{abstract}
In order to make the intelligent materials have a new development in the field of mechanical engineering, composition of intelligence materials is briefly introduced in this article; Design thought of intelligent materials are detailed analyzed; Main functions of the intelligent materials was described; Typical application of the intelligent materials are stated, optical fiber and shape memory alloy are used as a example. At last the research difficulties of energy conversion and storage of intelligent materials are detailed discussed, and the future research direction of intelligent materials is pointed out. In the future, the development direction of intelligent materials mainly include these aspects, such as the study of bionics theory on conceptual design of intelligent materials, the study of intelligence material intrinsic properties and evaluation system of intelligence quotient, the study of dissipative structure theory applied in intelligent materials, the principles of complex integration and design theory of intelligence materials, the nonlinear theory of integrated intelligent structure, the humanoid intelligent control theory, etc.
\end{abstract}

Keywords-sensitive materials; optical fiber; shape memory alloys; bionic technology; sensor

\section{INTRODUCTION TO INTELLIGENCE MATERIALS}

Intelligence material is called as sensitive material, and it is combined by sensor or sensing elements with traditional materials. Intelligence materials are intelligence characteristics of materials it can feel the stimuli from environmental then analysis, process, judgment, and to take certain measures to moderate response [1]. Intelligent materials have three essential elements follow as perception, drive and control.

The measure indicator of intelligence material general used of qualitative indicator, it is measured mainly from following aspects: the perception function of which can detect and discriminate outside of stimulus strength [2]; the drive function of which can response outside changes; it can according to set of way to select and control response; its reaction compared with sensitive, timely and appropriate; it can quickly to recovery to original state after external stimulus elimination [3]. The measure indicator should also take into account the unified between the strong function and low complexity from the use, therefore, a quantitative indicator is proposed, it called intelligence effect represented by MI, it mean the number of functions divided by the system components number of the n-th .

\section{INTELLIGENT MATERIAL DESIGN IDEAS}

Design ideals of intelligent materials are relative with the following factors: material development history, influence of artificial intelligence computer [4], intelligent materials manufacturing, software function material, the material aspirations, energy delivery, and materials with the timeline. Development and design ideas of intelligence materials should start from two different point of view, one is the bionic technology, another is structures characteristic of the intelligent material itself.

\section{A. Bionic technology}

Due to the organism has environmental perception and response, it provides a blueprint for intelligence material design [5]. Intelligence is a characteristic of organisms, and the environmental perception and response inspire people to explore the adaptive of the material systems and structures from the bio-science and bio-engineering and approximation to the multiple functions of the organism.

\section{B. Structure feature of intelligent material itself}

Intelligence material structure is found from the structure characteristics of the intelligent material. Mesoscope dimensions structure plays an extremely important role in the development of intelligent materials, and it is a size structure from nanometer to micron, such as the people most familiar with the proteins and enzymes. It has a very important physiological function, it belonging to the nano-size of structure material. 


\section{FUNCTIONS OF INTELLIGENT MATERIALS}

The design ideas of intelligent materials are multifunctional composite of materials and bionic design. Intelligence materials have sensing function, the feedback function, information recognition and accumulation function, response function, self-diagnosis function, selfrepair function and self-regulating function [6].

(1) Sensing function when used as a sensor: it is able to perceive the information from outside or their environmental conditions such as load, stress, strain, vibration, thermal, light, electromagnetic, chemical, nuclear radiation intensity and its variation.

(2) Feedback function when used as a feedback device: it can compare input information with output information of the system by sensing networks and provide the results to control systems timely and accurately.

(3) Information identification function and accumulation function: it can identify various types from sensing networks and accumulate the information.

(4) Response function: it has driven function, according to the external environment and internal conditions change, to make an appropriate response in time dynamically, select and control the response according to the setting of set way and take the necessary action.

(5) Self-diagnosis function: it can self-diagnose a series of issues such as system failures and misjudgments and correct the issues through comparison and analysis the current situation with the past situation of the system.

(6) Self-recovery function: it can fix some local damage or destruction after an external stimulus eliminated, and can be quickly restored to its original state through self-breeding, self-growth, in-situ composite mechanism.

(7) Self-adjusting functions: it can adjust its structure and function automatically in a timely and change their status and behavior, according to the changing external environment and conditions, so as to make the material system is always in an optimal way to proper response to external changes.

\section{APPLICATION OF TYPICAL INTELLIGENT MATERIALS}

Intelligent materials is a complex system of material, collection of materials, intelligent processing, implementation systems, control systems and sensing systems in one[7]. Intelligent materials mainly used in building area, aircraft manufacturing, medical field and military field. Typical intelligent materials mainly include: optical fiber, shape memory alloys, shape memory polymers, piezoelectric materials, magnetostrictive materials, electro-rheological materials and Polymer colloids, and the applications of optical fiber and shape memory alloy is more common.

\section{A. Application of optical fiber}

Optical fiber is a transmission tool using the principle of total reflection of light transmission in fibers made of glass or plastic [8]. The conduction losses of optical fiber are far below the electric wires, therefore, optical fiber applies to long distance information transmission.

(1) Classification of optical fibers. According to the transmission modes of light optical fiber can be divided into multi-mode fiber and single-mode optical fiber. The glass core the center in multi-mode fiber is thicker, multiple-modes of light can be transmitted. The center glass core for single-mode fiber is thin, and it can only transfer one mode of light, because of their small dispersion, so it applied to remote telecommunications. The former is optimization the optical fiber transmission frequency on a single wavelength of light; the latter is optimization optical fiber transmission frequency on two wavelengths of light. The optic fiber can divide into mutant and gradient type optical fiber according to refractive index distribution.

(2) Principle of optical fiber structure. Optical fiber is made of two different refractive index of glass, and the outer diameter is $0.1-0.2 \mathrm{~mm}$ [9]. The core refractive index of glass is larger than the outer glass $1 \%$. According to the principles of refraction and total reflection of light, when the angle light irradiates into the inner core and outer layer of interface is greater than the critical angle of total reflection, light passes through the interface and all reflection, in which case the interface through innumerable total reflection of light, to ragged core routes, spreading until the other end of the optical fiber. Autofocus type fiber is combined similar to those performed by many micro lenticular lens, forcing the rays converge to the center, thus refractive index in the central is the highest, then it is uniform attenuation along radial outward[10]. The causes of optical fiber decay mainly due to eigen, bending, extrusion, impurities, non-uniform, butt joint etc.

(3) Advantages of optical fiber transmission. Optical fiber transmission has the following advantages:

Band-width. The more width of optical frequency band, the greater of the transport capacity, bandwidth has reached $30000 \mathrm{GHz}$ in minimum loss region, make arrangements for 2000 optical carrier and accommodates a million of channels.

Low loss. The losses of optical fiber conduction are 100 million times smaller than the coaxial cable, so the transmission distance is much larger than coaxial cable.

Weight. A optical fiber cable in diameter is about $13 \mathrm{~mm}$ composed by 48 optical fibers, and coaxial cable diameter is $47 \mathrm{~mm}$, and because of low density of glass fiber, it has advantages such as small diameter, light weight, easy installation and so on.

High fidelity. Optical fiber transmission does not relay amplifier, so introduced a new nonlinear distortion will not enlarge link.

\section{B. Application of shape memory alloys}

(1) Shape Memory Effect. SME refers to material under conditions of temperature changes in the outside can change its shape and has a reversible change. SME divided into one way, two-way and full-way memory effect [11]. When heating it recovery high temperature phase shape; when cooling it can recovery cryogenic phase shape of phenomenon so it called two-way memory effect; when heating it recovery high temperature phase shape, and cooling becomes the same shape and orientation instead of cryogenic phase shape of phenomenon is called full memory effect. Alloy with shape memory function known as the shape memory alloy that is SMA.

(2) Work principle of shape memory alloys. Shape memory alloy is heated to a critical temperature above for shape memory heat treatment, formed martensite phase after rush cold, then exert a deformation, shape memory alloy is heated to critical temperature above again, makes 
crystal phase reverse shift, make the cryogenic martensite phase inverse becomes high temperature austenite phase (also known as parent phase) and recovery to inherent shape before deformation or return to martensite phase through internal elastic energy is released in follow-up cooling[12]. In the process of phase-change of shape memory alloys, rigid, resistance, friction and number of sound waves of materials are changed. Shape memory alloy is heated to a critical temperature above for shape memory heat treatment; Shape memory alloy is heated to critical temperature above again[13]. And because of the shape memory alloy heat shrink and phase change under external field, resulting in large deformation and stress, material can be strengthened. Using these features, shapememory material can be used as smart materials actuation device so that material is not only self-diagnosis, but with self-injury self-healing ability. In the process of phasechange of shape memory alloys, rigid, resistance, friction and number of sound waves of materials are changed.

(3) Fe-based shape memory alloys. Fe-based SMA is divided into two categories: based on the thermo elastic martensite phase change and based on non-thermo elastic reversible martensite phase change. Fe-based shape memory alloys have to meet the following conditions: Parent phase has high yield strength or low elastic limit; Martensite phase intersect causes a small change in volume and strain; Martensite analogy (c/a) and favor twinning substructure; The less Ms favor twinning substructure and increase the yield strength of the parent phase. Application of Fe-based shape memory alloys [14]. Fe-based shape based on the alloy provides a set of fitting pipe connection technology, its advantages are anticorrosion, good sealing, resistance to high pressure, strong

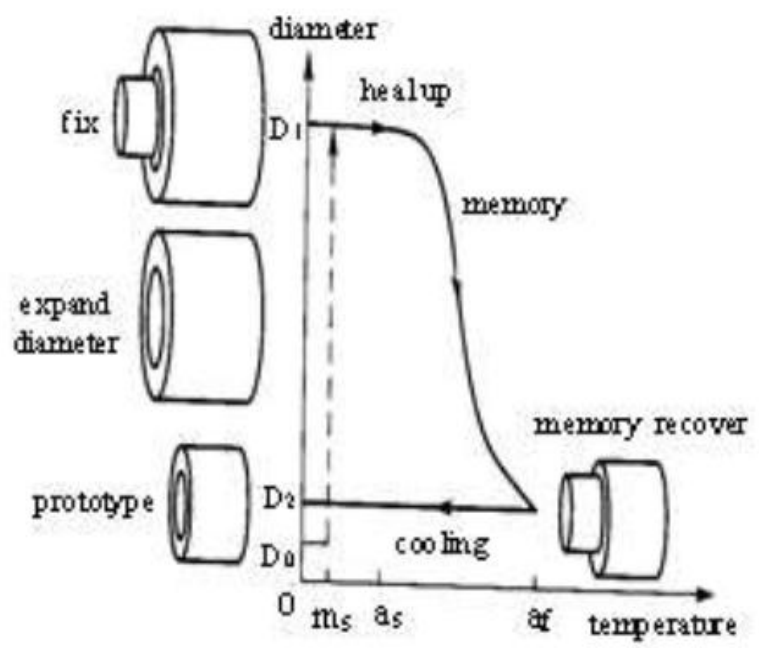

Figure1. Diagram of a shape memory alloy pipe joints connection etc. (shown as Fig .1).

(4) Cu-based shape memory alloys. Application of $\mathrm{Cu}-$ based shape memory alloys. First, it can be used as automatic fasteners. Use a memory alloy fitting, its inner diameter is smaller than the connection pipe outside diameter $4 \%$, expansion of pipe diameter under the low temperature, then set on the connected pipe [15]. When the tube temperature rises above as, it will shrink to the original shape, so as to connect the connected pipes securely (shown as Fig .1). SMAs is a thermo-sensitive materials, it can select appropriate alloy to control M s, Af, and temperature change range according to environmental conditions, to control the temperature by the shape memory of SMAs. The handle controller is shown in Fig .2.

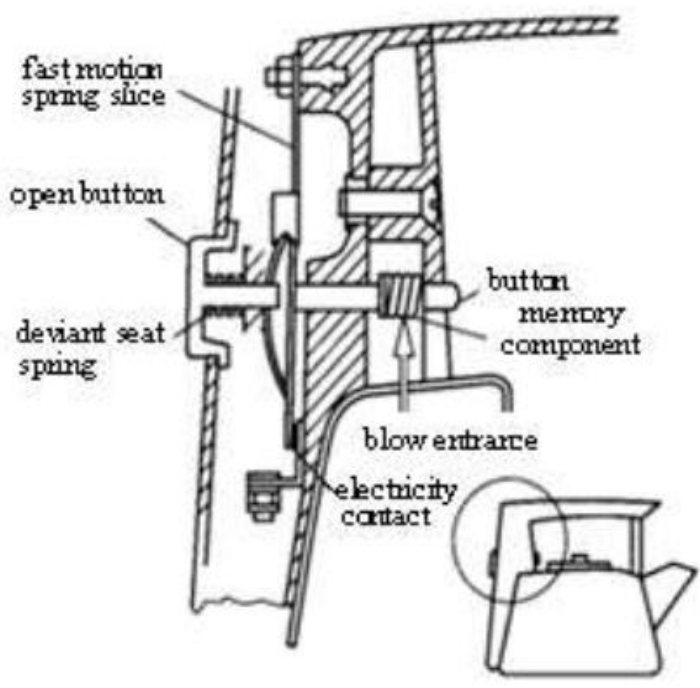

Figure2. Electrical heating kettle controller

(5) Ti-Ni base shape memory alloys. Ti-Ni based SMA in addition to its unique shape memory function, also has some excellent characteristics such as wear resistance, corrosion resistance, high damping and hyper-elastic. Ti$\mathrm{Ni}$ based SMA can meet the demand for all kinds of engineering and medical applications. Application of Ti-Ni shape memory alloys. Produce a radio communication antenna for shape memory. Ti-Ni shape memory intelligent magnetic material can be produced a spaceship's radio communication antenna, its production step as shown in Fig .3. First, Ti-Ni alloy wire is heated to $65{ }^{\circ} \mathrm{C}$, making it is translated into the austenite phase (Fig .3 (a)), and then alloy wire is cooled to below $65^{\circ} \mathrm{C}$ and translated into martensite phase [16]. Second, at room temperature martensite alloy wire is cut into small pieces, and then bent into a shape of antenna, small pieces of cross are welded fixed in antenna (Fig .3 (b)).Third, press the antenna into a small group, its volume is reduced to one-tenth, so that spaceships easy to carry (Fig .3 (c)). Last, when need to use the antenna, just heat the small group to $77^{\circ} \mathrm{C}$, so that martensite translated into austenite completely, and restoration the original size and shape completely (Fig .3 (d)). 


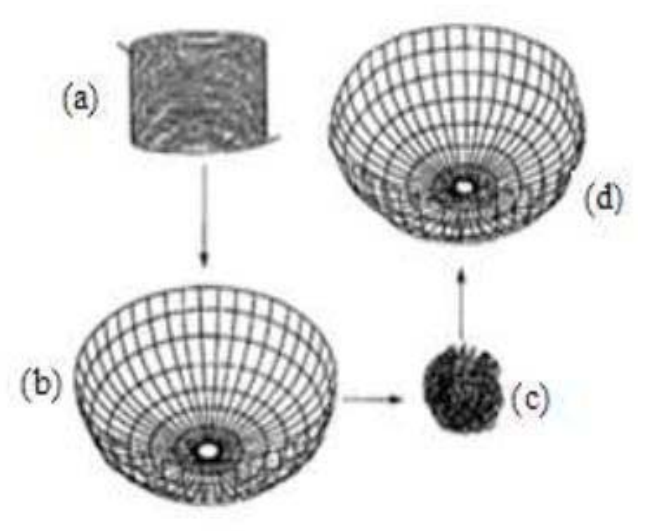

Figure3. Radio communication antenna

In difficult operating environment such as in a closed vacuum, using shape memory alloy fastening rivets to rivet constructional elements. Operation method as follow: First, make the rivet tail memory shape into opening, rivet tail is straightened in the dry ice before fastening, then insert rivet in the hole on fastener, the temperature rising produces shape recovery, the rivet tail is open to fasten (as shown in Fig .4). Ti-Ni shape memory alloys are widely used in the field of medicine, orthodontics, plastic surgery and so on.

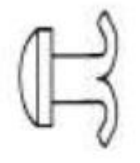

(a) Straige (D) W

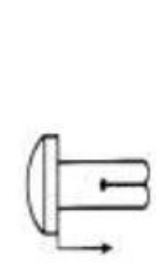

(b) Srieghis (1) (1)

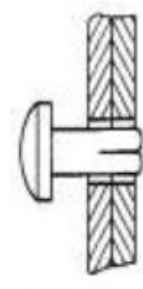

(a) hent (D)

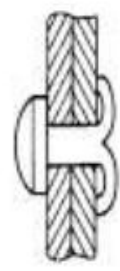

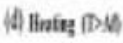

Figure4. Schematic diagram of shape memory alloy rivets fastened

\section{CONCLUSION}

Intelligence material is an open system that relies on continuous input energy or substance to dynamically adjust the adaptive capacity of the outside world from the surroundings to maintain its activity similar to organisms. Therefore, the primary research difficulty of intelligent materials is energy conversion and storage, and another difficulty is that upgrades ability of intelligent materials such as self-learning, self-judgment and self-upgrade.
Intelligence materials research focuses on the following aspects future: study of bionics theory on conceptual design of intelligent materials; study of intelligence material intrinsic properties and evaluation system of intelligence quotient; study of dissipative structure theory applied in intelligent materials; principles of complex integration and design theory of intelligence materials; nonlinear theory of integrated intelligent structure ; humanoid intelligent control theory.

\section{REFERENCES}

[1] Sun Yuxiu. The preparation methods and application of nanometer materials [M]. Beijing: China textile press, 2012

[2] Han Ming. The application and development trend of intelligent materials [J]. Henan building materials in henan, 2011 (3), pp. 147149.

[3] Hao Zhenhai, Wang Zhenzhong. Research progress of surface modification of nano powder [J]. Journal of chemical industry in guangdong. 2010 (5), pp. 13-14

[4] Nii Y,Arima T,Kim H Y,et al.Effect of Randomness on Ferroelastic Transitions:Disorder-Induced Hysteresis LooP Rounding in Ti-Nb-O Martensitic Alloy [J]. Physical Review B,2010,pp.214.

[5] Tang Shanfa. Gemini surfactant application of nanomaterials [M]. Beijing: chemical industry press, 2011

[6] Yang S,Bao H,Zhou C,et al.Large Magnetostriction form MorPhptropic Phase Boundary in Ferromagnets [J]. Physical Review Letters,2010(104),pp.197-201.

[7] Wang Dong,Wang zhiyun,Li Ju. With lron of material progress in computer simulation [J]. China's material progress, 2012 (31),pp. 8 -13 .

[8] Wang D,Wang Y,Zhang Z,et al. Modeling Abnormal Strain States in Ferroelastic Systems: the Role of Point Defects [J]. Physical Review Letters,2010(105) ,pp.205-702.

[9] Ren X,Wang Y,Zhou Y,et al.Strain Glass in Ferroelastic Systems:Premartensitic Tweed Versue Strain Glass[J]. Philosophical Magazine,2010,pp.141.

[10] Wang D, Zhang Z, Zhang J , et al.,Strain Glass in Fe-Doped Ti$\mathrm{Ni}[\mathrm{J}]$.Acta Materialia,2010,pp.6206.

[11] Sun Taolei. Electric bionic intelligent interface materials [J]. Scientific observation. 2010 (6),pp.60

[12] Qu Ping Bai Haolong, Wang Xuan. Cellulose based 3D nano materials surface modification [J]. China science and technology papers. 2013 (02), pp. 116-119

[13] Chen Yingjie, su-ling yao. Intelligent materials [M], Beijing: mechanical industry publishing house, 2013.

[14] Ni Lizhong. Composite materials science and engineering [M]. Beijing:Science press, 2011

[15] Yu Xiaoguang,Hu Mianhuai. Capacitor motor load sensor sensitive element research $[\mathrm{J}]$. Electric logging and instrumentation, 2012(533),pp.87-89.

[16] Wu Xueli. Carbon nanotube surface graft polymer modified [D]. Lanzhou university. 2010 